\title{
Third-generation prospect theory
}

\author{
Ulrich Schmidt • Chris Starmer • Robert Sugden
}

Published online: 14 May 2008

(C) Springer Science + Business Media, LLC 2008

\begin{abstract}
We present a new theory of decision under uncertainty: third-generation prospect theory $\left(\mathrm{PT}^{3}\right)$. This retains the predictive power of previous versions of prospect theory, but extends that theory by allowing reference points to be uncertain while decision weights are specified in a rank-dependent way. We show that $\mathrm{PT}^{3}$ preferences respect a state-conditional form of stochastic dominance. The theory predicts the observed tendency for willingness-to-accept valuations of lotteries to be greater than willingness-to-pay valuations. When $\mathrm{PT}^{3}$ is made operational by using simple functional forms with parameter values derived from existing experimental evidence, it predicts observed patterns of the preference reversal phenomenon.
\end{abstract}

Keywords Prospect theory · Preference reversal $\cdot$ Reference dependence

\section{JEL Classification D81}

In this paper we present a new theory of decision under uncertainty: third-generation prospect theory ( $\mathrm{PT}^{3}$ for short). The motivation for the theory is empirical: our model is presented as a descriptive theory intended to outperform the current 'best buys' in the literature. $\mathrm{PT}^{3}$ has three key features: reference dependence, decision weights and uncertain reference points (i.e. reference points that can be lotteries). The first two features are the common characteristics of different versions of prospect theory, including the original (or first-generation) version (Kahneman and

U. Schmidt

Department of Economics, Christian-Albrechts-Universität zu Kiel, 24098 Kiel, Germany

U. Schmidt

Kiel Institute for the World Economy, 24105 Kiel, Germany

C. Starmer $(\bowtie)$

School of Economics, University of Nottingham, Nottingham NG7 2RD, UK

e-mail: chris.starmer@nottingham.ac.uk

R. Sugden

School of Economics, University of East Anglia, Norwich NR4 7TJ, UK 
Tversky 1979) and the later cumulative (or second-generation) versions featuring rank-dependent decision weights (e.g. Starmer and Sugden 1989; Luce and Fishburn 1991; Tversky and Kahneman 1992; Wakker and Tversky 1993). Variants of cumulative prospect theory are increasingly widely applied in both theoretical and empirical work (recent examples are Davies and Satchell 2004; Trepel, Fox and Poldrack 2005; Wu, Zhang and Abdellaoui 2005; Baucells and Heukamp 2006; Schmidt and Zank 2008) and some have argued that such theories may be serious contenders for replacing expected utility theory at least for specific purposes (see Camerer 1989). No doubt this is partly because there is considerable empirical support for both reference-dependence and decision weights (see Starmer 2000).

First- and second-generation prospect theory have a common limitation: the reference points from which prospects are evaluated are assumed to be certainties. If reference points are interpreted as endowments or status quo positions, these theories cannot be applied to problems in which a decision maker is endowed with a lottery and has the opportunity to sell or exchange it. Such problems are common in real economic life-for example, buying insurance or selling stocks. They also feature in many experimental designs and, in consequence, a good deal is known about how in fact decision-makers respond to them. This evidence shows two particularly well-established and robust patterns of deviation from the predictions of conventional expected utility theory.

The first is that willingness-to-accept (WTA) valuations of lotteries tend to be greater than willingness-to-pay (WTP) valuations (e.g. Knetsch and Sinden 1984; Loomes, Starmer and Sugden 2003). Intuitively, one might expect that loss aversion, as modelled in prospect theory, would explain this effect; but because a WTA valuation of a lottery is made from a reference point at which the decision-maker is endowed with that lottery, that intuition cannot be expressed in existing versions of prospect theory.

The second deviation is preference reversal (PR). The classic instances of PR involve decisions relating to pairs of gambles. In the simplest cases, gambles are binary lotteries with just one positive outcome (the prize); the other outcome is zero. One of the lotteries, usually called the 'P bet', gives the better chance of winning a prize while the other, the ' $\$$ bet', has the larger prize. In a typical experiment, agents' preference orderings over pairs of such bets are elicited in two ways: in a pairwise choice task, and by comparing WTA valuations of lotteries elicited separately for P and $\$$ bets. There is a widely observed tendency for agents to reveal a preference for the $\mathrm{P}$ bet in choice but the $\$$ bet in valuation. We will call this pattern standard $P R$. Such inconsistencies between choice and valuation might arise through chance or error. But the opposite inconsistency, in which the $\$$ bet is chosen but the $\mathrm{P}$ bet is given a higher value (non-standard $P R$ ), is much less frequently observed. It is this asymmetry between the two types of reversal which constitutes the puzzle of PR. Because existing versions of prospect theory cannot deal with WTA valuations of lotteries, they cannot be applied to classic PR problems.

We have set ourselves the task of generalising cumulative prospect theory so that it can encompass uncertain reference points. Thus, $\mathrm{PT}^{3}$ inherits all the descriptive success (and, of course, any descriptive failure ${ }^{1}$ ) of cumulative prospect theory. As we shall show, it can also explain both PR and the WTA/WTP disparity for lotteries.

\footnotetext{
${ }^{1}$ Some descriptive limitations of prospect theory are discussed by Birnbaum and Bahara (2007).

Springer
} 
In all versions of prospect theory, the subjective value of a given monetary lottery, viewed from a given certain reference point, is a weighted average of the subjective values of the monetary gains or losses associated with the lottery outcomes. Gains and losses are defined relative to the reference point; an increasing value function (analogous with a utility function in expected utility theory) is used to transform these into positive or negative indices of subjective 'value'. These indices are then aggregated by using decision weights, which are determined by using a probability weighting function (a strictly increasing mapping from the interval $[0,1]$ onto itself) which assigns to each probability $p$ a transformed probability $w(p)$. In cumulative prospect theory, this function is used in a rank-dependent way, which we describe in more detail in Section 1. A zero gain is defined to have zero value, and so has no impact on the overall value of the lottery. Decision weights for gains are assigned cumulatively, beginning with the largest gain and working downwards. A mirrorimage method is used to assign weights to losses. As an illustration of this operation applied to gains, consider a lottery which gives monetary gains of zero, $£ 5$ and $£ 10$ with respective probabilities $0.5,0.3$ and 0.2 . The weight for the largest positive gain, $£ 10$, is determined by a direct transformation of the relevant probability, giving the weight $w(0.2)$. The decision weight for the $£ 5$ gain is then defined as $w(0.2+0.3)$ $-w(0.2)$. Notice that the sum of the decision weights for the $£ 5$ and $£ 10$ gains is $w(0.5)$, i.e. the transformation of the sum of the corresponding probabilities. This construction ensures that, for any two lotteries A, B, if A stochastically dominates B, then $\mathrm{A}$ is preferred to $\mathrm{B}$, viewed from any certain reference point.

A generalisation of this theory to uncertain reference points requires two components. The first is a definition of 'gain' and 'loss' relative to such reference points. Our approach, in the tradition of Savage (1954), is to define preferences over acts, where an act is an assignment of consequences to states of the world. A stochastic reference point is modelled as a particular act, the reference act. Gains and losses are defined separately for each state of the world. For example, consider a lottery with tickets numbered $1, \ldots, 100$; one ticket will be drawn at random and its owner will win $£ 100$. Consider an agent who is endowed with ticket 1 and who treats this as her reference act. She is offered the opportunity to exchange ticket 1 for ticket 2 . If she consents to this exchange, there is a 0.01 probability that ticket 1 will be drawn, in which case she will be $£ 100$ worse off than if she had kept her initial endowment. There is also a 0.01 probability that ticket 2 will be drawn, in which case she will be $£ 100$ better off. If any other ticket is drawn, she is neither better off nor worse off. Thus, the option of taking ticket 2 in exchange for ticket 1 , viewed from the reference act, gives $£ 100$ gain with probability $0.01, £ 100$ loss with probability 0.01 , and no change in wealth with probability 0.98 .

The second component for a generalisation is a method for determining decision weights when reference points are uncertain. Given our Savage-style framework, we need a rank-dependent method of assigning decision weights to any act $f$, viewed relative to any reference act $h$. For any state of the world $s$, we use $f(s)$ and $h(s)$ to denote the outcomes of $f$ and $h$ in that state. Our approach is to rank states in terms of the ex post net gain from choosing $f$ rather than $h$ - that is, by the value of $f(s)-h(s)$; separate rankings are constructed for 'gain' states (for which $f(s)-g(h)$ is positive) and 'loss' states (for which it is negative). These rankings are then used to determine decision weights, just as rankings of outcomes are used in second-generation prospect 
theory. This approach ensures that preferences respect a state-conditional form of stochastic dominance, even when the reference point is uncertain.

By using these two components, any parameterised form of second-generation prospect theory can be generalised to the case of uncertain reference points. No additional parameters are required. This is significant, because the empirical literature provides a lot of information about the parameterisations of prospect theory that are most successful in organising experimental data, and about the values that the various parameters typically take. Thus, we can investigate whether $\mathrm{PT}^{3}$ explains observations of behaviour in decision problems with uncertain reference points, using parameterisations whose empirical validity has already been established.

We show that $\mathrm{PT}^{3}$ predicts the observed WTA/WTP disparity for lotteries, given only the standard assumption of loss aversion in the value function. Perhaps more surprisingly, we show that, when $\mathrm{PT}^{3}$ is configured with parameterisations typical of those already established in the empirical literature, it predicts standard patterns of PR. Consequently we suggest that $\mathrm{PT}^{3}$ is a best buy theory: it offers the predictive power of previous variants of prospect theory and adds to that an explanation of PR. The latter comes 'free of charge' since it involves no extra parameters and no re-parameterisation.

\section{$1 \mathrm{PT}^{3}$ in its most general form}

In this section we introduce $\mathrm{PT}^{3}$. In this theory, preferences are defined over Savage acts. Consider a finite state space $S$, consisting of the states $s_{i}, i=1, \ldots, n$, and a set of consequences $X$ given by an interval of the real line. In interpreting the theory, we treat consequences as levels of wealth. As in the case of second-generation prospect theory, $\mathrm{PT}^{3}$ can be formulated either for risk or for uncertainty. In this paper, we restrict attention to risk. The extension to uncertainty is straightforward and is exactly as in second generation prospect theory. For decision making under risk each state $s_{i}$ has an objective probability $\pi_{i} \geq 0$, with $\Sigma_{i} \pi_{i}=1 . F$ is the set of all acts. A particular act $f \in F$ is a function from $S$ to $X$, i.e. an act $f$ specifies for each state $s_{i}$ the resulting consequence $f\left(s_{i}\right) \in X$.

As in other versions of prospect theory, preferences over acts are referencedependent. We formalise this following the approach of reference-dependent subjective expected utility theory (RDSEU), as proposed by Sugden (2003). For any three acts $f, g, h, f \succsim_{h} g$ denotes that $f$ is weakly preferred to $g$ viewed from $h$, the reference act. (The corresponding relations of strict preference and indifference are written as $f \succ_{h} g$ and $f \sim_{h} g$.) For present purposes the reference act can be interpreted as the status quo position. We depart from earlier versions of prospect theory by not requiring $h$ to be a constant act (i.e. an act which gives the same consequence in every state). Instead, we adopt a key innovation of RDSEU.

Sugden's axiom system implies maximisation of the function:

$$
V(f, h)=\Sigma_{i} v\left(f\left[s_{i}\right], h\left[s_{i}\right]\right) \pi_{i}
$$

In this expression, $v\left(f\left[s_{i}\right], h\left[s_{i}\right]\right)$ is a relative value function. It can be interpreted as the desirability of the consequence of act $f$ in state $s_{i}$ relative to the consequence of a reference act $h$ in the same state. This function is strictly increasing in its first 
argument, with $v\left(f\left[s_{i}\right], h\left[s_{i}\right]\right)=0$ when $f\left(s_{i}\right)=h\left(s_{i}\right)$. The function $V(f, h)$ is the expectation of relative value. It assigns a real value to any act $f \in F$ viewed from any reference act $h \in F$ (i.e. $V: F \times F \rightarrow \mathbb{R}$ ). It is a preference representation in the sense that, for all $f, h, g$ in $F, f \succsim_{h} g \Leftrightarrow V(f, h) \geq V(g, h)$.

In opting for the RDSEU approach, we have made a significant modelling decision. This approach is based on a state-contingent conception of reference-dependence. That is, gain/loss comparisons are made separately for each state of the world; thus, the pattern of gains and losses associated with any act $f$, viewed from any reference act $h$, depends on the state-contingent juxtaposition of consequences in $f$ and $h$. An alternative approach, proposed by Köszegi and Rabin (2006, 2007), takes no account of state-contingency. Instead, reference-dependent preferences are defined over prospects (i.e. probability distributions over consequences). Notice that two acts can have different assignments of consequences to states of the world, and thus be distinct acts, while inducing the same probability distribution over consequences and so being represented by a single prospect. Implicitly, the Köszegi-Rabin (KR) approach treats such acts as equivalent to one another. It specifies the expected relative value of any prospect $A$, viewed from any (certain or uncertain) reference prospect $\mathrm{R}$, in such a way as to coincide with the implications of the RDSEU specification (Eq. 1) in the special case in which A and R are stochastically independent. ${ }^{2}$ Since this condition holds trivially when $\mathrm{R}$ is a degenerate prospect, the KR and RDSEU approaches coincide when reference points are certain.

However, the KR approach has paradoxical implications. Consider any two nondegenerate acts $f$ and $h$ that induce the same probability distribution of consequences A. What is the preference ranking of $f$ and $h$, viewed from $h$ ? The KR approach treats this problem as if $f$ and $h$ were stochastically independent. If those acts were in fact independent, moving from $h$ to $f$ would induce gains in some states and losses in others. These gains and losses exactly offset one another in money terms, but if preferences are loss-averse (as is assumed in most versions of prospect theory), the subjective impact of the losses outweighs that of the gains. Expressed in the language of RDSEU, we have $h \succ_{h} f$, which makes intuitive sense if the acts really are perceived as stochastically independent. In fact, there is experimental evidence of such preferences: subjects who have been endowed with numbered lottery tickets are typically unwilling to exchange these for equivalent but differently numbered tickets, even when offered small payments for doing so (Bar-Hillel and Neter 1996). ${ }^{3}$ But in the KR framework, this result has to be expressed in terms of reference-dependent preferences over prospects. If $\mathrm{A}$ is the prospect that represents the acts $h$ and $f$, we reach the conclusion that $\mathrm{A}$ is strictly preferred to $\mathrm{A}$, viewed from $\mathrm{A}$, with the apparent implication that the agent has a strict preference for 'keeping' some lottery rather than 'exchanging' it for exactly the same lottery. One way to avoid such paradoxical conclusions would be to interpret the KR theory as assuming that each lottery under evaluation is perceived by the agent as stochastically independent of the reference

\footnotetext{
${ }^{2}$ Compare Eq. 1 above with Eq. 2 in Köszegi and Rabin (2007).

${ }^{3}$ In Bar-Hillel and Neter's 'Experiment 2', subjects are unwilling to exchange lottery tickets even if they know that the number of the winning ticket will not be announced. This suggests that subjects use a statecontingent conceptualisation of gains and losses even if they will never know which state is realised.
} 
point. On this interpretation, the KR theory is less general than RDSEU, but yields the same conclusions in those cases to which both apply.

The preference representation in Eq. 1, like that proposed by KR, is linear in probabilities. $\mathrm{PT}^{3}$ relaxes this restriction of RDSEU by generalizing Eq. 1 to:

$$
V(f, h)=\Sigma_{i} v\left(f\left[s_{i}\right], h\left[s_{i}\right]\right) W\left(s_{i} ; f, h\right)
$$

where $W\left(s_{i} ; f, h\right)$ is the decision weight assigned to state $s_{i}$ when $f$ is being evaluated from $h$. In principle, decision weights could be determined by a simple transformation of state probabilities (i.e. $\left.W\left(s_{i} ; f, h\right)=w\left(\pi_{i}\right)\right)$ as in Handa (1977). However, in the contemporary literature on prospect theory it has become conventional to construct decision weights cumulatively using a rank-dependent transformation (Quiggin 1982; Starmer and Sugden 1989; Tversky and Kahneman 1992). One of the key theoretical rationales for the cumulative construction is that, unlike first-generation prospect theory (which included some elements of Handa's approach), it results in monotonic preferences. That is, if one prospect stochastically dominates another, the first is preferred to the second when viewed from any certain reference point. In $\mathrm{PT}^{3}$ we retain the rank-dependent approach, but reconfigure it to work with state-contingent reference-dependence.

Given that we have chosen to follow the RDSEU approach to the definition of reference-dependence, there is little remaining freedom of manoeuvre for choosing how to specify rank-dependent decision weights. In order to construct cumulative weights for a given $f, h$ pair, states must be ordered according to the 'attractiveness' of $f$ 's consequences in each state. In a cumulative construction, the weight attached to a given state depends not only on the probability of that state but also on the position of its consequence in the ranking of all consequences associated with $f$. To ensure that monotonicity is preserved when probabilities are transformed, it is essential that the 'attractiveness' ranking is determined by the function whose decision-weighted expected value represents preferences. In RDSEU, this is the relative value function. Thus, the position of each state $s_{i}$ in the ranking must be determined by the ranking of $v\left(f\left[s_{i}\right], h\left[s_{i}\right]\right)$ values. This implies that the ordering of states must be constructed separately for each $f, h$ pair. Further, if we are to generalise cumulative prospect theory, we must have separate rank-dependent transformations of probability for gains and losses, and these transformations must be mirror-images of one another.

Consider any $f, h$ pair. Relative to that pair, there is a weak gain in a state $s_{i}$ if $f\left(s_{i}\right) \geq h\left(s_{i}\right)$, and a strict loss if $f\left(s_{i}\right)<h\left(s_{i}\right)$. Let $m^{+}$be the number of states in which there are weak gains and let $m^{-}=n-m^{+}$be the number of states in which there are strict losses. We re-assign subscripts so that, for all subscripts $i, j$, we have $i>j$ if and only if $v\left(f\left[s_{i}\right], h\left[s_{i}\right]\right) \geq v\left(f\left[s_{j}\right], h\left[s_{j}\right]\right)$, and so that the states with weak gains are indexed $m^{+}, \ldots, 1$ and the states with strict losses are indexed $-1, \ldots,-m^{-}$.

Cumulative decision weights are then defined as follows:

$$
W\left(s_{i} ; f, h\right)=\left\{\begin{array}{cc}
w^{+}\left(\pi_{i}\right) & \text { if } i=m^{+}, \\
w^{+}\left(\Sigma_{(j \geq i)} \pi_{j}\right)-w^{+}\left(\Sigma_{(j>i)} \pi_{j}\right) & \text { if } 1 \leq i \leq m^{+}-1, \\
w^{-}\left(\Sigma_{(j \leq i)} \pi_{j}\right)-w^{-}\left(\Sigma_{(j<i)} \pi_{j}\right) & \text { if }-m^{-}+1 \leq i \leq-1 \\
w^{-}\left(\pi_{i}\right) & \text { if } i=-m^{-},
\end{array}\right.
$$


where $w^{+}$and $w^{-}$are, respectively, probability weighting functions for the gain and loss domains (i.e. $w^{+}, w^{-}$are strictly increasing mappings from $[0,1]$ onto $[0,1]$ ). This specification has two important implications. First, if $h$ is a constant act, Eq. 3 implies the same assignment of decision weights as in cumulative prospect theory. Thus, the monotonicity property of cumulative prospect theory carries over to $\mathrm{PT}^{3}$. That is, for all acts $f, g$, and for all constant acts $h$, if the probability distribution of consequences induced by $f$ stochastically dominates that induced by $g$, then $f$ is strictly preferred to $g$, viewed from $h$. Second, a state-contingent form of monotonicity holds for all reference acts. For any acts $f$, $g$, we will say that $f$ statewise dominates $g$ if $f\left(s_{i}\right) \geq g\left(s_{i}\right)$ for all states $s_{i}$, with a strict inequality for at least one state with non-zero probability. It follows from Eqs. 2 and 3 that if $f$ statewise dominates $g$, then for all reference acts $h, f \succ_{h} g$.

$\mathrm{PT}^{3}$, as specified by Eqs. 2 and 3, straightforwardly captures several models as special cases. RDSEU is the special case in which decision weights are untransformed state probabilities (i.e. $w^{+}\left(\pi_{i}\right)=w^{-}\left(\pi_{i}\right)=\pi_{i}$ for all $i$ ). Cumulative prospect theory is the special case in which the relative value function takes the form $v\left(f\left[s_{i}\right], h\left[s_{i}\right]\right)=u\left(f\left[s_{i}\right]-\right.$ $h\left[s_{i}\right]$ ), where $u($.$) is a 'value' function, { }^{4}$ and in which reference acts are constrained to be certainties (i.e. $h\left(s_{i}\right)=h\left(s_{j}\right)$ for all $\left.i, j\right)$. Expected utility theory is the special case in which decision weights are untransformed state probabilities, as in RDSEU, and relative value is independent of the reference outcome (i.e. $v\left(f\left[s_{i}\right], h\left[s_{i}\right]\right)=u\left(f\left[s_{i}\right]\right)$ where $u($.$) is a von Neumann-Morgenstern utility function).$

\section{A parameterised form of $\mathrm{PT}^{3}$}

One way of evaluating $\mathrm{PT}^{3}$ as a descriptive theory is to test its novel predictionsthat is, the predictions it makes about behaviour in decision problems with uncertain reference points - using parameterisations whose validity has been established for earlier versions of prospect theory. This approach requires that we select specific functional forms for our general model. In doing this we are guided by three criteria. First, we seek a model flexible enough to allow us to investigate how decisionmaking behaviour varies with three key aspects of the agent's preferences: attitudes to consequences, attitudes to probability, and attitudes to gain and loss. Second, subject to that constraint, we seek to use the simplest model possible - that is, a model with just one parameter for each of the three attitudes we consider. Third, for comparability with existing evidence, we use wherever possible the functional forms that are most common in previously published research.

We begin by imposing the restriction that the relative value function takes the form $v\left(f\left[s_{i}\right], h\left[s_{i}\right]\right)=u(z)$, where $z=f\left(s_{i}\right)-h\left(s_{i}\right)$. When $h$ is a constant act, this special case of state-contingent reference-dependence is equivalent to that built into earlier generations of prospect theory; $u($.$) is the counterpart of the value function in those$ theories.

\footnotetext{
${ }^{4}$ The restriction $v\left(f\left[s_{i}\right], h\left[s_{i}\right]\right)=u\left(f\left[s_{i}\right]-h\left[s_{i}\right]\right)$ prevents cumulative prospect theory from taking account of income effects. Kahneman and Tversky (1979, pp. 277-278) comment that 'strictly speaking', value should be defined as a function in two arguments - changes in wealth relative to a current asset position, and that position itself. The simpler function they use is presented as 'a satisfactory approximation'.
} 
Next, we specify a functional form for $u($.$) . We adopt the power function which$ has been widely used in recent empirical literature (see Starmer 2000). Specifically,

$$
u(z)=\left\{\begin{array}{l}
z^{a} \text { if } z \geq 0 \\
-\lambda|z|^{a} \text { if } z<0 .
\end{array}\right.
$$

The parameters $\alpha$ and $\lambda$ are required to be strictly positive. The first of these parameters controls the curvature of the value function. If $\alpha<1$, this function is concave in the domain of gains and convex in the domain of losses (the property of diminishing sensitivity). Diminishing sensitivity imparts a tendency for risk aversion with respect to gains and risk-loving with respect to losses. While the empirical literature has suggested some differences in the exponents of the value function between the domains of gains and losses, in the interests of parsimony we will apply the same exponent in both domains. The parameter $\lambda$ controls attitudes to gain and loss. With $\lambda=1$ there is loss neutrality. For $\lambda$ values above unity, there is loss aversion: losses are weighted more heavily than gains. For values below unity, the opposite is the case.

We model decision weights by means of a single-parameter probability weighting function. Again, for reasons of parsimony we impose the restriction of identical weighting functions for gains and losses (i.e. $w^{+}(\pi)=w^{-}(\pi)$ ). Hence for the purpose of parameterisation the probability weighting function is denoted simply by $w(\pi)$; it takes the form

$$
w(\pi)=\pi^{\beta} /\left(\pi^{\beta}+[1-\pi]^{\beta}\right)^{1 / \beta}
$$

with $\beta>0$. This type of weighting function has been discussed by Tversky and Kahneman (1992) and Prelec (1998); variants of it have been widely used in the empirical literature. With $\beta=1$, decision weights are linear (i.e. $w(\pi)=\pi$ ) but for values of $\beta$ less than 1 and going down to around 0.4 , the function generates an inverse-S pattern of weights with over-weighting (under-weighting) of probabilities below (above) some critical probability $\pi^{*}$. Inverse-S weighting has been reported across a wide range of empirical studies (Wu and Gonzalez 1996, 1999; Abdellaoui 2000; Bleichrodt and Pinto 2000; Abdellaoui, Vossmann and Weber 2005).

That completes the specification of the generic model to be used in the calibrations. We will refer to this specification as parameterised $P T^{3}$.

Notice that, when applied to cases in which reference acts are certainties, parameterised $\mathrm{PT}^{3}$ can also be interpreted as a parameterisation of cumulative prospect theory. In fact, models of this kind have already been estimated using experimental data (e.g. Tversky and Kahaneman 1992; Loomes, Moffatt and Sugden 2002). Thus, parameter values from these estimations are applicable to our model.

We take the following to be relatively well-established stylised facts concerning the median values of the three parameters for experimental subjects. First, many studies suggest the existence of loss aversion, while its opposite is almost unknown; values of the loss aversion parameter in the range $1 \leq \lambda \leq 2.5$ would capture a reasonably wide range of evidence. Studies fitting variants of prospect theory with power utility almost invariably find diminishing sensitivity. Although some studies have found values of $\alpha$ as low as 0.22 (Loomes, Moffatt and Sugden 2002, note 17), 
values in the range $0.5 \leq \alpha \leq 1$ are typical. Inverse-S probability weighting, while not universal, is a very common finding; it would be reasonable to expect values of $\beta$ in the range $0.5 \leq \beta \leq 1$. These ranges of values will be the focus for evaluating the predictions of our model.

\section{Explaining WTA/WTP disparities for lotteries}

We now show that parameterised $\mathrm{PT}^{3}$ implies that WTA valuations of lotteries exceed WTP valuations whenever $\lambda>1$. This is not a surprising prediction: intuitively, one might expect the WTA/WTP disparity to be explained by loss aversion, which is represented in our model by the parameter restriction $\lambda>1$. Nevertheless, as we pointed out in Section 1, previous versions of prospect theory have not been able to make this prediction. Further, it is significant that our model makes a clear-cut prediction about WTA/WTP disparities despite the complications introduced by decision weights.

The hypothesis that individuals tend to prefer to retain status quo positions rather than to move away from them can be expressed in terms of the non-reversibility of reference-dependent preferences. ${ }^{5}$ We will say that an agent's preferences are nonreversible if, for all acts $f$ and $g, f \succsim_{g} g \Rightarrow f \succsim_{f} g$; they are strictly non-reversible if $f \succsim_{g} g \Rightarrow f \succ_{f} g$. Intuitively, suppose the agent is endowed with $g$ and is willing to exchange this for $f$. Non-reversibility implies that if she makes this exchange, and if her reference point adjusts to $f$, she does not then have a strict preference for reversing the exchange. Given the assumptions built into parameterised $\mathrm{PT}^{3}$, strict non-reversibility implies the WTA/WTP disparity. To see why, let $g$ be any act, let $h$ be a constant act which gives consequence $z$ in every state, and suppose that $h \sim_{g} g$. Then $z$ is the WTA valuation of $g$. Let $h^{\prime}$ be a constant act, giving $z^{\prime}$ in every state, such that $h^{\prime} \sim_{h^{\prime}} g$. If income effects are zero, as implied by the assumptions of parameterised $\mathrm{PT}^{3}, h^{\prime}$ is the WTP valuation of $g$. If preferences are strictly nonreversible, the supposition $h \sim_{g} g$ implies $h \succ_{h} g$ : the agent would not be willing to pay $z$ to get $g$. Thus, by virtue of the monotonicity properties of $\mathrm{PT}^{3}$, the WTP valuation of $g$ is less than the WTA valuation.

We now show that parameterised $\mathrm{PT}^{3}$ satisfies strict (weak) non-reversibility if $\lambda>$ 1 (if $\lambda \geq 1$ ). Consider any two acts $f$ and $g$. Let $F$ be the set of states $s_{i}$ such that $f\left(s_{i}\right)>$ $g\left(s_{i}\right)$ and let $G$ be the set of states $s_{j}$ such that $g\left(s_{j}\right)>f\left(s_{j}\right)$. Combing Eq. 2 with the parameterisation of the relative value function:

$$
\begin{gathered}
V(f, g)=\Sigma_{i \in F}\left(f\left[s_{i}\right]-g\left[s_{i}\right]\right)^{\alpha} W\left(s_{i} ; f, g\right)-\Sigma_{j \in G} \lambda\left(g\left[s_{j}\right]-f\left[s_{j}\right]\right)^{\alpha} W\left(s_{j} ; f, g\right) \\
V(g, f)=\Sigma_{j \in G}\left(g\left[s_{j}\right]-f\left[s_{j}\right]\right)^{\alpha} W\left(s_{j} ; g, f\right) \\
-\Sigma_{i \in F} \lambda\left(f\left[s_{j}\right]-g\left[s_{j}\right]\right)^{\alpha} W\left(s_{i} ; g, f\right)
\end{gathered}
$$

\footnotetext{
${ }^{5}$ This idea was introduced by Tversky and Kahmenan (1991), and developed by Munro and Sugden (2003), to characterise reference-dependence in preferences over multi-dimensional consumption bundles.
} 
The specification of decision weights in Eq. 3 implies $W\left(s_{i} ; f, g\right)=W\left(s_{i} ; g, f\right)$ for all $i \in F$ and $W\left(s_{j} ; f, g\right)=W\left(s_{j} ; g, f\right)$ for all $j \in G$. Hence: ${ }^{6}$

$$
V(f, g)+V(g, f)=(1-\lambda)\left(\Sigma_{i \in F}\left[f\left(s_{i}\right)-g\left(s_{i}\right)\right]^{\alpha} W\left[s_{i} ; f, g\right]+\Sigma_{j \in G}\left[g\left(s_{j}\right)-f\left(s_{j}\right)\right]^{\alpha} W\left[s_{j} ; g, f\right]\right) .
$$

The right-hand side of Eq. 8 is positive, zero, or negative according to whether $\lambda$ is less than, equal to, or greater than unity. Thus, the parameter restriction $\lambda>1$ implies $V(f, g)+V(g, f)<0$ or, equivalently, $f \succsim_{g} g \Rightarrow_{\mathrm{f}} \succ_{f} g$, i.e. strict non-reversibility. Similarly, $\lambda \geq 1$ implies weak non-reversibility.

\section{Explaining preference reversal}

In this section, we apply parameterised $\mathrm{PT}^{3}$ to preference reversal experiments. ${ }^{7}$ For simplicity, we restrict attention to $\mathrm{P}$ and $\$$ bets that give either a positive payoff or zero. This case has been widely studied in the empirical literature. Consider two acts with this structure. Specifically, let $f^{\mathrm{P}}$ represent an act giving an increment of wealth $\mathrm{x}$ with probability $p$ and a zero increment otherwise, and let $f^{\$}$ be an act giving an increment of wealth $y$ with probability $q$ and a zero increment otherwise, with $y>x>0$ and $1>p>$ $q>0$. As a normalisation, we define consequences as increments or decrements of wealth relative to the agent's wealth (treated as a certainty) prior to the PR experiment. This preexperimental endowment is denoted by the constant act $h$, where $h\left(s_{i}\right)=0$ for all $i$.

A feature of the power utility function used in parameterised $\mathrm{PT}^{3}$ is that model predictions are unchanged if all outcomes are multiplied by any positive constant. Exploiting this property, we may normalise the expected value of the $\mathrm{P}$ bet to unity by setting its payoff $x=1 / p$. Given this normalisation, we can characterise any pair of $\mathrm{P}$ and $\$$ bets by a three-parameter vector $(p, q, r)$, where $p$ is the probability of winning the prize in the $\mathrm{P}$ bet, $q$ is the corresponding probability for the $\$$ bet, and $r$ is the expected value of the $\$$ bet as a ratio of the expected value of the $\mathrm{P}$ bet (implying that the positive payoff of the $\$$ bet is $y=r / q$ ). Notice that the condition $y>$ $x$ (i.e. the $\$$ bet has the higher prize) implies $r p>q$.

Consider an agent choosing between the two bets in the PR experiment. Her reference point is the constant act $h$. Using Eqs. 2, 3 and 4, the agent's choice between the two bets is determined by: ${ }^{8}$

$$
f^{P} \succsim_{h} f^{\S} \Leftrightarrow w(p)(1 / p)^{\alpha} \geq w(q)(r / q)^{\alpha} ;
$$

the values of $w(p)$ and $w(q)$ are given by Eq. 5 .

\footnotetext{
${ }^{6}$ Notice that the derivation of this result uses two symmetries between the treatments of gains and losses that are specific to our parameterisation: the weighting function is the same for gains and losses, and the exponent of $u($.$) is the same for gains and losses.$

${ }^{7}$ Our analysis of PR does not depend on the cumulative transformation of probabilities. The acts that we analyse have no more than one strictly positive consequence and no more than one strictly negative one. For such acts, the cumulative transformation is observationally equivalent to Handa's simple transformation.

${ }^{8}$ In addition, $f^{\mathrm{P}} \sim{ }_{h} f^{\S} \Leftrightarrow w(p) / w(q)=(p r / q)^{a}$. From now on, to avoid cluttering the exposition, we will not state conditions for indifference explicitly. In all cases, the condition for indifference can be constructed from the condition for weak preference by substituting an equality for a weak inequality.
} 
Now consider the agent's WTA valuations of the bets. Take the case of the P bet. The agent is endowed with this bet and is asked to consider selling it. Her situation is depicted by the following matrix (in which the columns are states, with probabilities shown at the top, the rows are acts, and the entries in the cells are consequences):

\begin{tabular}{lll}
\hline & $p$ & $1-p$ \\
\hline$h^{\mathrm{P}}$ & $1 / p$ & 0 \\
$g^{\mathrm{P}}$ & $z_{\mathrm{P}}$ & $z_{\mathrm{P}}$ \\
\hline
\end{tabular}

Her reference act, denoted $h^{\mathrm{P}}$, is the P bet. Her WTA valuation of this bet, denoted $z_{\mathrm{P}}$, is the increment of wealth such that she is indifferent between retaining $h^{\mathrm{P}}$ or giving up $h^{\mathrm{P}}$ in exchange for the certainty of that increment. Hence, we define $z_{\mathrm{P}}$ as the sure payoff of some constant act $g^{\mathrm{P}}$ defined such that $V\left(g^{\mathrm{P}}, h^{\mathrm{P}}\right)=0$. With $z_{\$}$ defined in an analogous way, the values of $z_{\mathrm{P}}$ and $z_{\$}$ are then determined, respectively, by the solutions to equations

$$
w(1-p) z_{P}^{\alpha}-w(p) \lambda\left([1 / p]-z_{P}\right)^{\alpha}=0
$$

and

$$
w(1-q) z_{\$}^{\alpha}-w(q) \lambda\left([r / q]-z_{\$}\right)^{\alpha}=0 .
$$

Substituting in the parameterisation of $w($.$) , the solutions of these equations are:$

$$
z_{P}=(1 / p) /\left([(1-p) / p]^{\beta / \alpha}(1 / \lambda)^{1 / \alpha}+1\right)
$$

and

$$
z_{\$}=(r / q) /\left([(1-q) / q]^{\beta / \alpha}(1 / \lambda)^{1 / \alpha}+1\right)
$$

It is easy to see that the preference ranking of the bets, as given by Eq. 9, need not be the same as the ranking of their WTA valuations, as given by Eqs. 12 and 13. A sufficient condition for the two rankings to be the same, and hence for PR not to occur, is the combination of parameter values $\lambda=1, \alpha=1$ and $\beta=1$, implying that both choice and valuation are determined by the maximisation of expected monetary value. In general, however, differences between the two rankings can be induced by asymmetric attitudes to gain and loss (i.e. $\lambda \neq 1$ ), non-linearity of the value function (i.e. $\alpha \neq 1$ ), or non-linearity of the probability weighting function (i.e. $\beta \neq 1$ ).

To see that attitudes to gain and loss are relevant, it is sufficient to notice that $\lambda$ appears in both Eqs. 12 and 13 but not in Eq. 9. To see that the shape of the probability weighting function is relevant, it is sufficient to notice that the terms $w(1-$ $p$ ) and $w(1-q)$ appear in Eqs. 10 and 11 respectively, but not in Eq. 9. To see that the curvature of the value function is relevant, notice that the choice and valuation expressions use different points on this function. For example, in the case of the $\mathrm{P}$ bet (for which the prize is $1 / p$ ), the choice expression includes the term $(1 / p)^{\alpha}$, whereas the valuation expression includes the terms $z_{\mathrm{P}}{ }^{\alpha}$ and $\left([1 / p]-z_{\mathrm{P}}\right)^{\alpha}$.

Given all this, it would not be surprising to find (as is in fact the case) that some combinations of parameter values induce standard PR, that is, the conjunction of $f^{\mathrm{P}} \succ$ $f^{\$}$ and $z_{\mathrm{P}}<z_{\$}$. But our objective is to do much more than this: it is to explore whether our model provides an empirically convincing account of observed instances of PR. 
We therefore need to ask whether standard PR is predicted when the model is calibrated using empirically-established values of $\lambda, \alpha$ and $\beta$.

It is convenient to explore the implications of the model graphically in $(\alpha, \lambda)$ space. This space is divided into quadrants by the lines $\alpha=1$ and $\lambda=1$. The stylised facts presented in Section 3 suggest that we should focus on the north-west quadrant, in which the value function is either linear or exhibits diminishing sensitivity (i.e. $\alpha \leq 1$ ) and in which there is either loss neutrality or loss aversion (i.e. $\lambda \geq 1$ ). We call this the empirically plausible quadrant.

For any given pair of bets and any given value of the decision weight parameter $\beta,(\alpha, \lambda)$ space can be divided into four regions by identifying two boundaries. One boundary - the choice boundary - identifies the locus of $(\alpha, \lambda)$ pairs along which the $\mathrm{P}$ and $\$$ bets are indifferent in choice (i.e. $f^{\mathrm{P}} \sim_{\mathrm{h}} f^{\$}$ ). A second boundary-the valuation boundary - is the locus of $(\alpha, \lambda)$ pairs along which the $\mathrm{P}$ and $\$$ bets have equal WTA valuations.

The following property of the choice boundary is an immediate implication of Eq. 9:

Property 1 The choice between $\mathrm{P}$ and $\$$ is independent of the value of $\lambda$. For any given value of $\beta$, there is a critical value of $\alpha$ at which the two bets are indifferent. At lower values of $\alpha, \mathrm{P}$ is chosen; at higher values, $\$$ is chosen.

This property reflects the fact that, in the choice task, all consequences are positive or zero. Thus, loss aversion has no role in determining choice. Because the negative domain of the value function is not relevant for this task, diminishing sensitivity (i.e. $\alpha<1$ ) plays essentially the same role in $\mathrm{PT}^{3}$ as diminishing marginal utility does in expected utility theory: the lower the value of $\alpha$, the greater the attractiveness of the safer $\mathrm{P}$ bet relative to the riskier $\$$ bet.

An examination of the expression for $z_{\mathrm{P}} / z_{\$}$, derived from Eqs. 12 and 13, yields the following property of WTA valuations:

Property 2 As $\lambda$ increases, the value of $z_{\mathrm{P}} / \mathrm{z}_{\$}$ falls; in the limit, as $\lambda \rightarrow \infty$, this value tends to $q / r p$, where $q / r p<1$.

In other words, increases in the loss aversion parameter $\lambda$ increase $z_{\$}$ relative to $z_{\mathrm{P}}$; at sufficiently high values of $\lambda$, we have $z_{\$}>z_{\mathrm{P}}$. Intuitively, this is because the act of selling a bet carries the risk of losing the prize of that bet in the state in which the bet wins; since the $\$$ bet has the higher prize, the potential for loss in selling it is greater. Thus, loss aversion induces a particular reluctance to sell the $\$$ bet.

Properties 1 and 2 are enough to give a preliminary sense of some of the combinations of parameter values that will induce PR. In order for the $\mathrm{P}$ bet to be selected in the choice task, $\alpha$ must be lower than some critical value. Given any such value of $\alpha$, the $\$$ bet will have the higher WTA valuation if the value of $\lambda$ is sufficiently high. Thus, standard PR is induced by the combination of sufficiently low $\alpha$ and sufficiently high $\lambda$.

Figure 1 plots the choice and valuation boundaries for a typical pair of bets, defined by $(p, q, r)=(0.8,0.2,1)$, with $\beta=1$. This particular combination of parameters will be called the benchmark case. Standard PR occurs in the region above the valuation boundary and to the left of the choice boundary; non-standard PR occurs in the region below the valuation boundary and to the right of the choice 


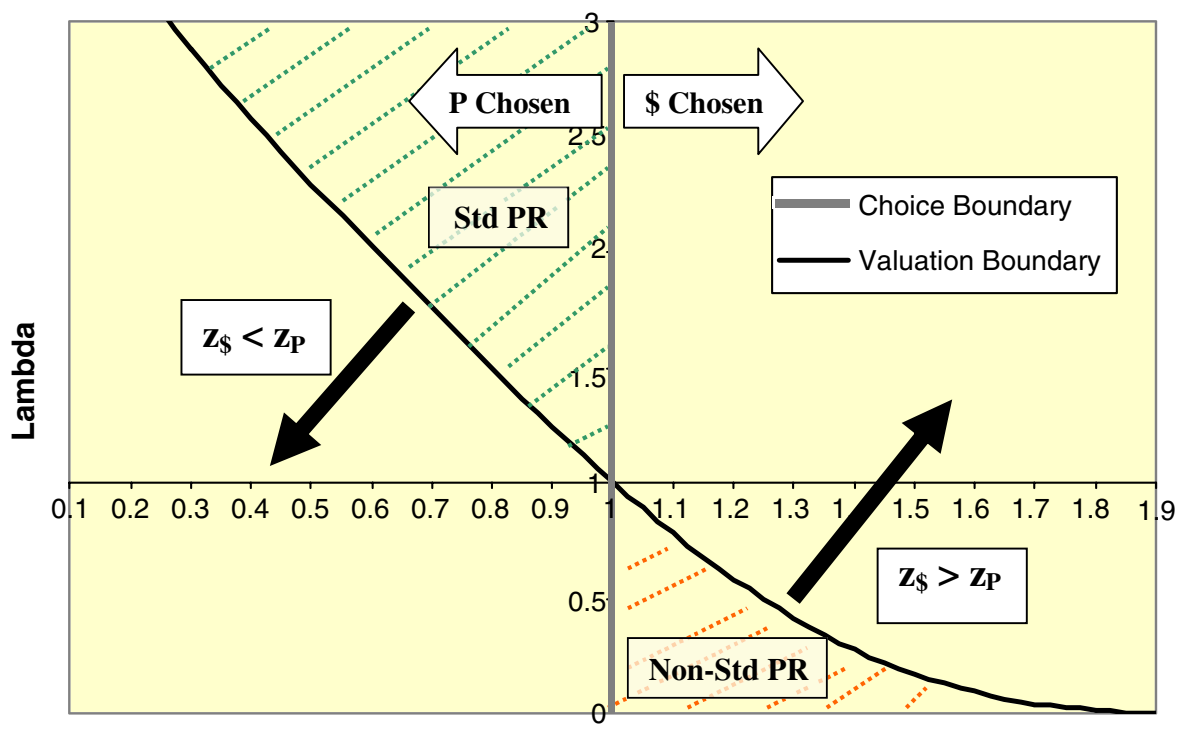

Alpha

Fig. 1 The choice and valuation boundaries $(p=0.8, q=0.2, r=1$, beta $=1)$

boundary. The two boundaries intersect at $(1,1)$. (This reflects the fact that, when $\alpha=1$, $\lambda=1$ and $\beta=1$, our model reduces to the maximisation of expected value; since the two bets have equal expected value, they are equally preferred and have equal valuations.) In this benchmark case, the empirically plausible quadrant is made up of two sub-regions, separated by the valuation boundary. Above this boundary there is standard PR. Below it, the P bet is both preferred in the straight choice and valued more highly. Thus, for the benchmark case, our model predicts the classic asymmetry between standard and non-standard reversals: the former occur at parameter values within the empirically plausible quadrant, while the latter do not.

It is easy to see that this qualitative conclusion is unaffected by changes in the values of the probability parameters $p$ and $q$ (given the defining condition $p>q$ ). Whenever $r=1$ and $\beta=1$, the choice and valuation boundaries intersect at $(1,1)$. Thus, $\mathrm{P}$ is chosen everywhere in the empirically plausible quadrant, ruling out the possibility of non-standard PR. There is always a non-empty region of this quadrant, to the left of the choice boundary and above the valuation boundary, in which standard PR occurs.

We now consider the effect of changes in the value of $r$ (the expected value of the $\$$ bet as a ratio of that of the $\mathrm{P}$ bet). Figure 2 plots the choice and valuation boundaries for $r=0.8, r=1.2$ and $r=1.4$ when the other parameters take their benchmark values (i.e. $p=0.8, q=0.2, \beta=1$ ). As $r$ increases, both boundaries shift to the left, expanding both the region in which the $\$$ bet is chosen and the region in which it has the higher valuation. The intuition for this is straightforward: an increase in $\mathrm{r}$ increases the $\$$-bet prize relative to the $\mathrm{P}$-bet prize, and so makes the $\$$ bet relatively more attractive. Notice that, at all values of $r$, standard PR occurs at some points in the empirically plausible quadrant, while non-standard PR occurs 


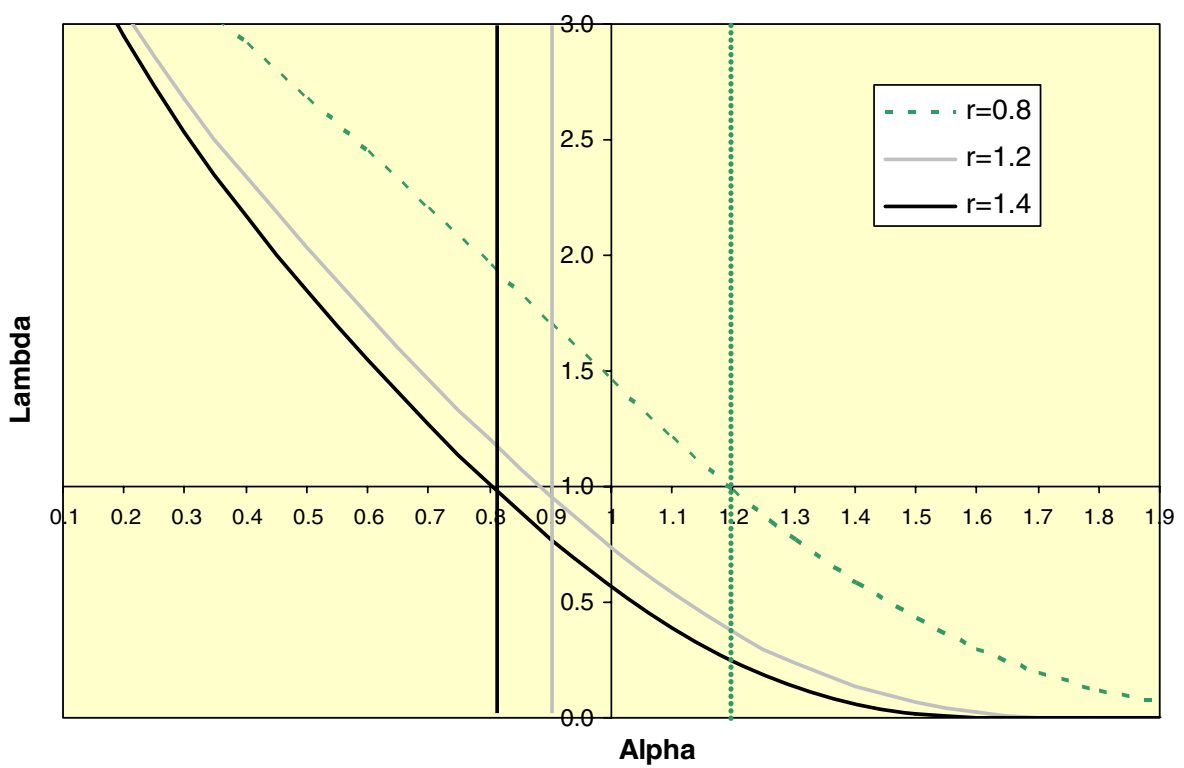

Fig. 2 Variation in $r(p=0.8, q=0.2$, beta $=1)$

only outside that quadrant. If $r>1$, the empirically plausible quadrant is made up of three sub-regions. Below the valuation boundary, the $\mathrm{P}$ bet is favoured in both choice and valuation. To the right of the choice boundary, the $\$$ bet is favoured in both choice and valuation. Above the valuation boundary and to the left of the choice boundary, there is standard PR. ${ }^{9}$

In the cases we have considered so far, our model has consistently predicted the classic asymmetry between standard and non-standard PR in the empirically plausible quadrant. However, it has failed to predict another stylised fact about PR experiments: that, even when the two bets have equal expected value, a significant proportion of subjects not only value the $\$$ bet more highly but also choose it in preference to the $\mathrm{P}$ bet. Because of the role of diminishing sensitivity in choice, our model predicts that $\mathrm{P}$ will be chosen whenever $r=1, \alpha<1$, and $\beta=1$. To show that this is not a problem for our approach, we note that the benchmark assumption $\beta=1$ is an extreme case - the case in which the probability weighting function is linear. We now consider the implications of assuming lower values of $\beta$, that is, an inverse$\mathrm{S}$ function.

\footnotetext{
${ }^{9}$ The reader may wonder why, in all the cases represented in Fig. 2, the valuation boundary intersects the choice boundary at $\lambda=1$. It can be shown that this property is induced by a special feature of the benchmark case, namely that the $\mathrm{P}$ and $\$$ bets are symmetrical in the sense that $q=1-p$. If the bets are symmetrical and if $\beta=1$, the valuation boundary passes through the point $(\ln [p / q] / \ln [r p / q], 1)$. Whether the bets are symmetrical or not, this point lies on the choice boundary. (Since this result has little substantive significance, we leave the proof to sufficiently curious readers.) If the assumption of symmetry is relaxed, the two boundaries may intersect at positive or negative values of $\lambda$. If $r>1$ and if the intersection is at a positive value of $\lambda$, there is a (typically small) region of the empirically plausible quadrant at which non-standard PR occurs.
} 


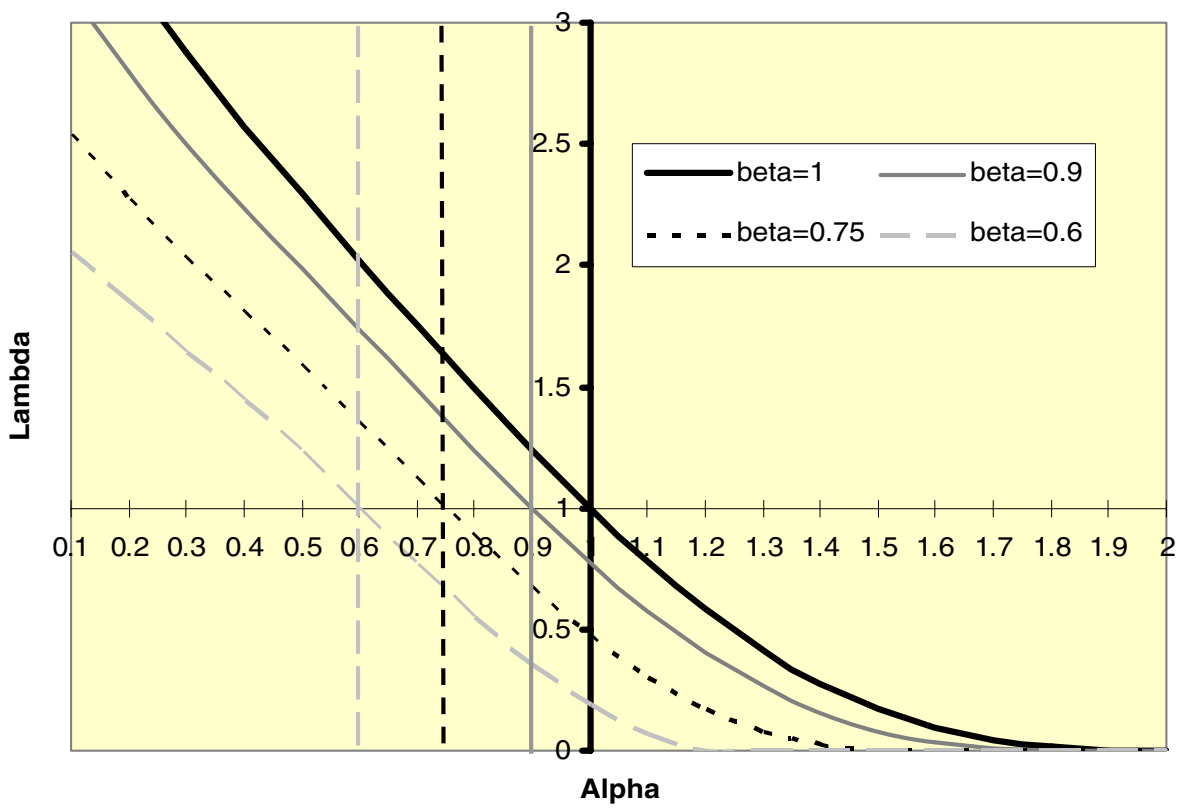

Fig. 3 Variation in beta $p=0.8, q=0.2, r=1$

Figure 3 plots the choice and valuation boundaries for three empirically plausible values of $\beta$, namely $0.9,0.75$ and 0.6 . The other parameters take their benchmark values (i.e. $p=0.8, q=0.2, r=1$ ). Essentially, the effect of reducing the value of $\beta$ is to shift both boundaries to the left, expanding the regions in which the $\$$ bet is favoured. The intuition for this is that, as the value of $\beta$ falls, small probabilities (such as 0.2 , the probability that the $\$$ bet wins) are increasingly overweighted while large probabilities (such as 0.8 , the probability that the $\mathrm{P}$ bet wins) are increasingly underweighted. The resulting configurations of choice and valuation boundaries are similar to those generated by setting $r>1$. Again, the empirically plausible quadrant of $(\alpha, \lambda)$ space is made up of three sub-regions. In one, the $\mathrm{P}$ bet is favoured in both choice and valuation; in another, the $\$$ bet is favoured in both choice and valuation; in the third, there is standard PR. ${ }^{10}$

All the diagrams we have presented so far have the common feature that standard PR occurs only when $\lambda>1$, and non-standard PR occurs only when $\lambda<1$. The reader should not infer from this that loss aversion is essential if $\mathrm{PT}^{3}$ is to predict standard PR. To the contrary, both standard and non-standard PR are compatible with $\lambda=1$ for some pairs of bets. Figures 4 and 5 illustrate these possibilities for, respectively, the

\footnotetext{
${ }^{10}$ The reader may have noticed that, in all three cases, the choice and valuation boundaries intersect at $(\beta$, 1). On the assumption that $r=1$, it can be shown that, for all admissible values of $p$ and $q$, the valuation boundary passes through $(\beta, 1)$. The choice boundary passes through the same point if and only if $q=1-$ $p$ (compare note 9). Figures 4 and 5, discussed in the next paragraph, illustrate some cases in which this symmetry condition does not hold.
} 


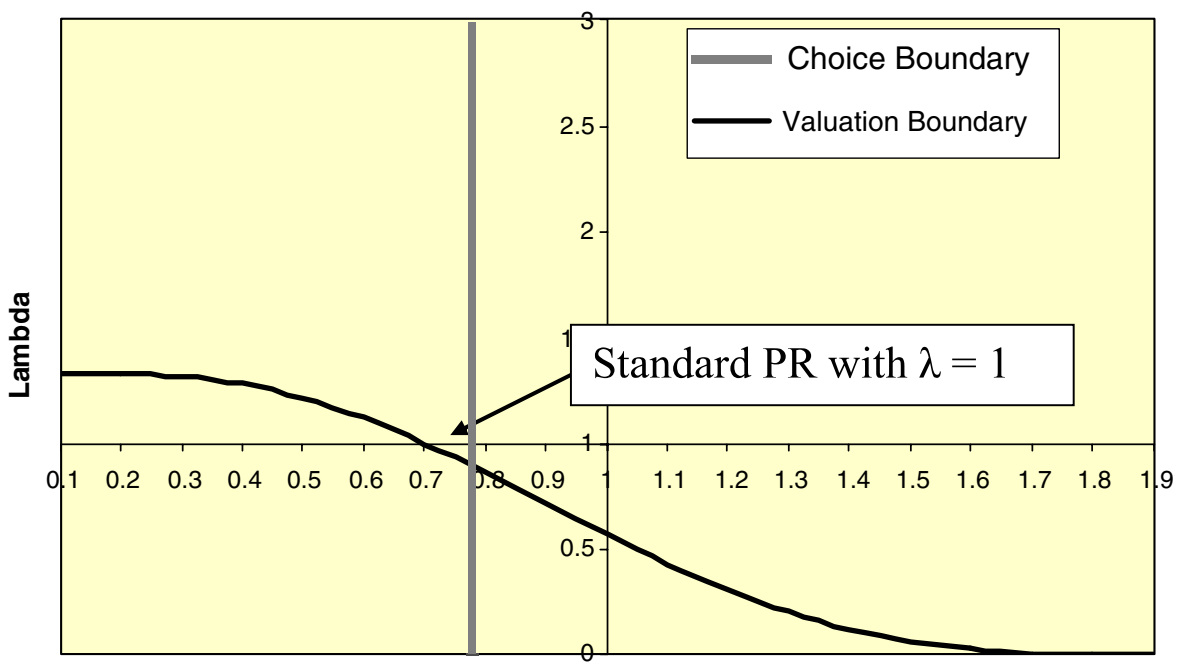

Alpha

Fig. 4 Bets $(0.8,0.4,1)$ with beta $=0.7$

pairs of bets $(0.8,0.4,1)$ and $(0.6,0.2,1)$ with $\beta=0.7 .{ }^{11}$ Conversely, $\mathrm{PT}^{3}$ can predict standard PR in cases in which the model differs from expected utility theory only in respect of loss aversion. (Consider the case in which $p=0.8, q=0.2, r=0.8$ and $\beta=1$, shown in Fig. 2. Notice that standard PR occurs at some points at which $\alpha=1$ and $\lambda>1$.) But these cases depend on special assumptions about the characteristics of the two bets. In contrast, the classic PR phenomenon occurs across a wide range of values of $p, q$ and $r$. Because our model includes the effects of loss aversion, diminishing sensitivity and inverse-S probability weighting, it is able to explain PR across the range in which it has been observed.

A further feature of the PR phenomenon has been identified by Tversky, Slovic and Kahneman (1990). Consider any pair of $\mathrm{P}$ and $\$$ bets faced by any given agent. Suppose that standard PR occurs: the P bet is strictly preferred in the choice task, but the $\$$ bet has the higher WTA valuation. Let $g^{\mathrm{P}}$ and $g^{\$}$ be the constant acts defined so that, for all states $s_{i}, g^{\mathrm{P}}\left(s_{i}\right)=z_{\mathrm{P}}$ and $g^{\$}\left(s_{i}\right)=z_{\$}$ (where, as before, $z_{\mathrm{P}}$ and $z_{\$}$ are the WTA valuations of the respective bets). Now consider the ranking of the four acts $f^{\mathrm{P}}, f^{\$}, g^{\mathrm{P}}$, $g^{\$}$, viewed from the constant reference act $h$, where $h\left(s_{i}\right)=0$ for all $s_{i}$. The occurrence of standard PR implies $f^{\mathrm{P}} \succ_{h} f^{\$}$ and (on the assumption that preferences are monotonic) $g^{\$} \succ_{h} g^{\mathrm{P}}$. How can these apparently conflicting preferences be reconciled? One possibility is that $f^{P} \succ_{h} g^{\mathrm{P}}$. That is, given a pairwise choice between the $\mathrm{P}$ bet and a certainty equal to its WTA valuation, the agent would strictly prefer the bet. If this is the case, the agent is said to have underpriced the $\mathrm{P}$

\footnotetext{
${ }^{11}$ In each case, the valuation boundary passes through $(\beta, 1)$, illustrating the general result stated in note 10. In general, if $\beta<1$ and $r=1$, the choice boundary lies to the right of (respectively passes through, lies to the left of) the point $(\beta, 1)$ if $p+q$ is greater than (equal to, less than) 1 . The proof of this result is omitted for brevity.
} 


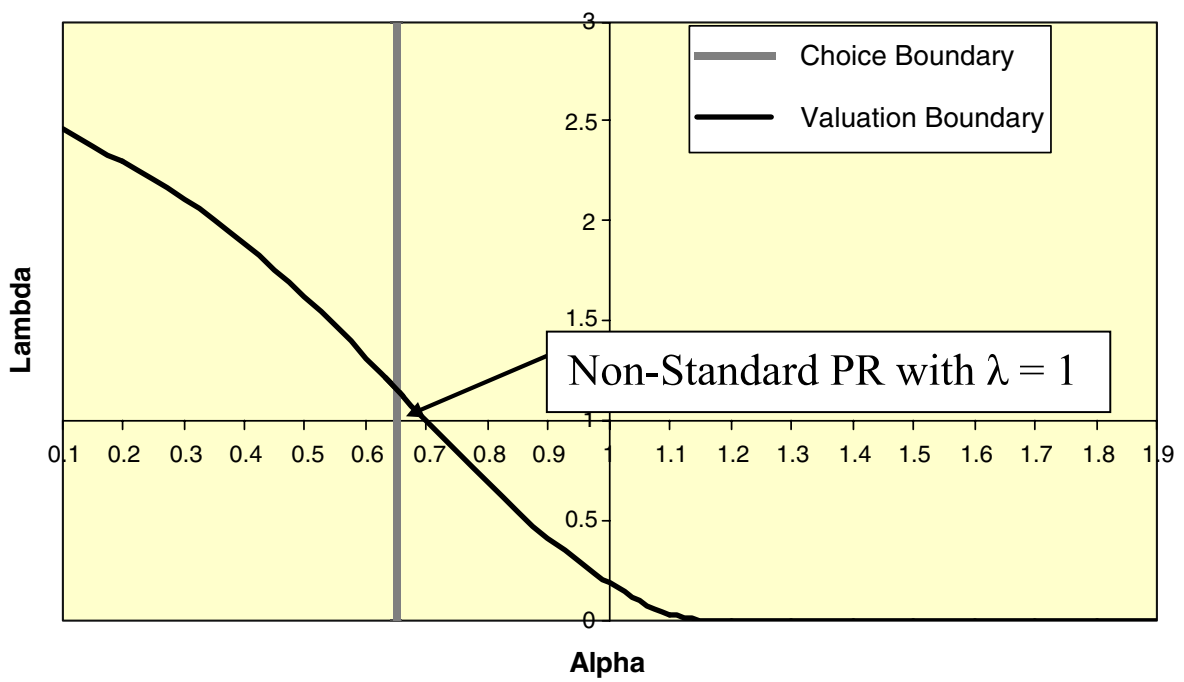

Fig. 5 Bets $(0.6,0.2,1)$ with beta $=0.7$

bet. A second possibility (not incompatible with the first) is that $g^{\$} \succ_{h} f^{\$}$. That is, in a pairwise choice between the $\$$ bet and a certainty equal to its WTA valuation, the agent would strictly prefer the certainty. If this is so, the $\$$ bet has been overpriced. If neither of these possibilities is the case, we have $f^{\mathrm{P}} \succ_{h} g^{\mathrm{P}}, g^{\mathrm{P}} \succsim_{h} f^{\mathrm{P}}, f^{\mathrm{P}} \succ_{h} f^{\$}$, and $f^{\$} \succsim_{h} g^{\$}$, which is a violation of transitivity. Tversky et al. report an experiment designed to discriminate between these possibilities; they find that standard PR is most commonly associated with overpricing of the $\$$ bet.

This observation is consistent with $\mathrm{PT}^{3}$. At the parameter values that induce standard PR, our model predicts overpricing of both the $\$$ and $\mathrm{P}$ bets. We show this for the $\$$ bet; the analysis for the $\mathrm{P}$ bet is essentially the same. The $\$$ bet is overpriced if it is strictly preferred to the certainty of $z_{\$}$, viewed from $h$. This is the case if and only if $z_{\$}{ }^{\alpha}>w(q)(r / q)^{\alpha}$, which can be rearranged as

$$
(1-w[q]) z_{\$}^{\alpha}-w(q)\left([r / q]^{\alpha}-z_{\$}^{\alpha}\right)>0
$$

Combining this inequality with Eq. 10, the equation which defines $z_{\$}$, the condition for overpricing is:

$$
(1-w[q]) z_{\$}^{\alpha}-w(q)\left([r / q]^{\alpha}-z_{\$}^{\alpha}\right)>w(1-q) z_{\$}^{\alpha}-w(q) \lambda\left([r / q]-z_{\$}\right)^{\alpha} .
$$

As one would expect, the LHS and RHS of Eq. 15 are equal (and equal to zero) if $\lambda=1, \alpha=1$ and $\beta=1$ (implying $w(q)=q$ and $w(1-q)=1-q$ ). In this case, $z_{\$}=r$, i.e. the WTA valuation of the bet is its expected monetary value, and there is neither underpricing nor overpricing.

Now consider the implications of variations in the agent's attitude to gain and loss. It is easy to see that, if $\alpha$ and $\beta$ are held constant at the values $\alpha=1$ and $\beta=1$, the inequality (Eq. 15) is satisfied if and only if $\lambda>1$. In other words, loss aversion induces overpricing. Alternatively, consider the implication of variations in the 
agent's attitude to consequences. If $\lambda$ and $\beta$ are held constant at $\lambda=1$ and $\beta=1$, Eq. 15 is satisfied if and only if $\alpha<1 .{ }^{12}$ Thus, diminishing sensitivity induces overpricing. Finally, consider variations in the agent's attitude to probability. If $\lambda$ and $\alpha$ are held constant at $\lambda=1$ and $\alpha=1$, Eq. 15 is satisfied if and only if $\beta<1 .{ }^{13}$ Thus, inverse-S probability weighting induces overpricing.

Bringing these results together, $\mathrm{PT}^{3}$ explains $\mathrm{PR}$ as a result of the interaction of empirically plausible degrees of loss aversion, diminishing sensitivity and probability weighting. The same attitudes induce the 'overpricing' effect observed by Tversky et al.

\section{Conclusions}

We have presented a new theory of choice under uncertainty: third generation prospect theory $\left(\mathrm{PT}^{3}\right) . \mathrm{PT}^{3}$ retains the empirically grounded features of previous variants of prospect theory (loss aversion, diminishing sensitivity and non-linear probability weighting), but extends that theory by allowing reference points to be uncertain. The resulting theory retains all the predictive power of those previous variants, but in addition provides a framework for determining the money valuation that an agent places on a lottery. We have shown that $\mathrm{PT}^{3}$ predicts the observed tendency for willingness-to-accept (WTA) valuations of lotteries to be greater than willingness-to-pay (WTP) valuations. More surprisingly, when $\mathrm{PT}^{3}$ is made operational by using simple functional forms with parameter values derived from existing experimental evidence, it predicts observed patterns of preference reversal (PR) across a wide range of specifications of $\mathrm{P}$ and $\$$ bets, consistent with the range in which that phenomenon has in fact been observed.

$\mathrm{PR}$ is one of the most notorious anomalies in individual decision making, but despite the large volume of literature it has generated, no satisfactory preference-based account of it has thus far been produced. In the psychology literature it has been common to interpret PR as evidence that preferences do not satisfy procedural invariance but, instead, depend upon the method used to elicit them. On this view, if preferences are to be invoked at all in explaining PR, those preferences must be context-sensitive: that is, they must allow different preferences to govern decisions in choice and valuation tasks. In the economics literature, various models of context-free preferences have been proposed as possible accounts of PR. One approach is to relax the independence and/ or reduction axioms of expected utility theory (Holt 1986; Karni and Safra 1987; Segal 1988). Subsequent studies, however, have generated strong PR in experimental designs implementing controls for the explanations postulated in these theories (Tversky, Slovic and Kahneman 1990; Cubitt, Munro and Starmer 2004). Another possible explanation is that PR arises as a consequence of context-free, but nontransitive preferences. Persistent non-transitive cycles of choice analogous to PR have been observed in experimental studies (Loomes, Starmer and Sugden 1989, 1991; Humphrey 2001), but the only preference theory that has been put forward to explain

\footnotetext{
${ }^{12}$ If $\lambda=1$ and $\beta=1$, Eq. 11 implies $0<z_{\$}<r / q$, while Eq. 15 reduces to $\left([r / q]-z_{\$}\right)^{a}>(r / q)^{a}-z_{\$}{ }^{a}$.

${ }^{13}$ If $\lambda=1$ and $a=1$, Eq. 15 reduces to $1-w[q]>w(1-q)$. This inequality holds if and only if $\beta<1$.

Springer
} 
such behaviour is regret theory (Bell 1982; Loomes and Sugden 1983), which has failed other tests (Starmer and Sugden 1998). A third approach is to explain PR by supplementing a conventional theory of preferences with some mechanism of stochastic error; but this has been shown to be empirically unconvincing (Schmidt and Hey 2004). Against this unpromising background, it is remarkable to find that observed patterns of PR are predicted by a simple extension of an existing and empirically well-supported preference-based theory.

We do not claim that $\mathrm{PT}^{3}$ provides a complete explanation of $\mathrm{PR}$. We recognise that psychologists have proposed credible non-preference mechanisms of contextsensitive choice and valuation behaviour that are consistent with observations of PR. Predictions based on those mechanisms have been tested and confirmed in experimental tasks other than PR and, in some cases, outside the domain of theories of choice under uncertainty (Slovic, Griffin and Tversky 1990). This evidence clearly suggests that non-preference mechanisms contribute to PR. We assert only that $\mathrm{PT}^{3}$ has a similar claim to be a model of mechanisms which contribute to that phenomenon. It too is based on psychologically credible hypotheses - loss aversion, diminishing sensitivity, the overweighting of small probabilities and the underweighting of large ones. It too is consistent with observations of PR. It too has been tested and confirmed in experimental tasks other than PR - namely, pairwise choices between lotteries involving gains and losses. If one accepts prospect theory as an explanation of observed regularities in choice among lotteries, it seems reasonable to infer that the mechanisms modelled by $\mathrm{PT}^{3}$ play a significant role in the explanation of PR.

We should also acknowledge that we have treated the use of WTA valuations as one of the defining characteristics of a PR experiment. Insofar as it relies on the assumption of loss aversion, the explanation of $\mathrm{PR}$ provided by $\mathrm{PT}^{3}$ is specific to such valuations. In fact, there have been surprisingly few experiments in which WTP valuations of $\mathrm{P}$ and $\$$ bets have been used. Such experiments have produced mixed results, but asymmetric PR is generally less pronounced than in WTA experiments, and sometimes is not present at all. It seems that WTP treatments tend to reduce the frequency of standard reversals and to increase the frequency of non-standard ones (Lichtenstein and Slovic 1971; Knez and Smith 1987; Casey 1991). These findings are compatible with the hypothesis that PR is the product of several causal mechanisms, at least one of which is in some way linked to WTA valuations. We suggest that $\mathrm{PT}^{3}$ captures a mechanism of the latter kind.

More generally, we offer $\mathrm{PT}^{3}$ as a natural extension of prospect theory-a theory that is already widely accepted as empirically successful. By allowing reference points to be uncertain, we have filled a major gap in that theory's previous domain of application. The result is a flexible and parsimonious model of choice under uncertainty which organises a large body of experimental evidence. We hope that it will find fruitful applications in future work.

Acknowledgements The authors thank Serge Blondel, Louis Lévy-Garboua, William Neilson, Peter Wakker, Horst Zank and anonymous referees for helpful comments on earlier drafts. We have also benefitted from discussions with participants at various conferences and seminars where we have presented this work. Sugden's work was supported by the Economic and Social Research Council (award no. RES 05127 0146). 


\section{References}

Abdellaoui, Mohammed. (2000). "Parameter-Free Elicitation of Utilities and Probability Weighting Functions," Management Science 46, 1497-1512.

Abdellaoui, Mohammed, Frank Vossmann, and Martin Weber. (2005). "Choice-Based Elicitation and Decomposition of Decision Weights for Gains and Losses under Uncertainty," Management Science 51, 1384-1399.

Bar-Hillel, Maya, and Efrat Neter. (1996). "Why Are People Reluctant to Exchange Lottery Tickets?" Journal of Personality and Social Psychology 70, 17-27.

Baucells, Manel, and Franz H. Heukamp. (2006). "Stochastic Dominance and Cumulative Prospect Theory," Management Science 52, 1409-1423.

Bell, David. (1982). "Regret in Decision Making under Uncertainty," Operations Research 20, 961-981.

Birnbaum, Michael H., and Jeffrey P. Bahra. (2007). "Gain-Loss Separability and Coalescing in Risky Decision Making," Management Science 53, 1016-1028.

Bleichrodt, Han, and Jose L. Pinto. (2000). "A Parameter-Free Elicitation of the Probability Weighting Function in Medical Decision Analysis," Management Science 46, 1485-1496.

Camerer, Colin F. (1989). “An Experimental Test of Several Generalized Utility Theories,” Journal of Risk and Uncertainty 2, 61-104.

Casey, Jeff. (1991). "Reversal of the Preference Reversal Phenomenon," Organizational Behavior and Human Decision Processes 48, 224-251.

Cubitt, Robin P., Alistair Munro, and Chris Starmer. (2004). "Testing Explanations of Preference Reversal," Economic Journal 114, 709-726.

Davies, Greg B. and Stephen E. Satchell. (2004). “Continuous Cumulative Prospect Theory and Individual Asset Allocation," Cambridge Working Papers in Economics (CWPE 0467).

Handa, Jagdish. (1977). "Risk, Probabilities, and a New Theory of Cardinal Utility," Journal of Political Economy 85, 97-122.

Holt, Charles A. (1986). "Preference Reversals and the Independence Axiom," American Economic Review 76, 508-515.

Humphrey, Stephen J. (2001). "Non-Transitive Choice: Event-Splitting Effects or Framing Effects?" Economica 68, 77-96.

Kahneman, Daniel, and Amos Tversky. (1979). "Prospect Theory: an Analysis of Decision under Risk," Econometrica 47, 263-291.

Karni, Edi, and Zvi Safra. (1987). "Preference Reversals and the Observability of Preferences by Experimental Methods," Econometrica 55, 675-685.

Knetsch, Jack, and John A. Sinden. (1984). "Willingness to Pay and Compensation Demanded: Experimental Evidence of an Unexpected Disparity in Measures of Value," Quarterly Journal of Economics 99, 507-521.

Knez, Marc, and Vernon Smith. (1987). "Hypothetical Valuations and Preference Reversals in the Context of Asset Trading." In A. Roth (ed), Laboratory Experimentation in Economics: Six Points of View. Cambridge: Cambridge University Press.

Köszegi, Botond, and Matthew Rabin. (2006). “A Model of Reference-Dependent Preferences,” Quarterly Journal of Economics 121, 1133-1165.

Köszegi, Botond, and Matthew Rabin. (2007). "Reference-Dependent Risk Attitudes," American Economic Review 97, 1047-1073.

Lichtenstein, Sarah, and Paul Slovic. (1971). "Reversals of Preferences between Bids and Choices in Gambling Decisions," Journal of Experimental Psychology 89, 46-55.

Loomes, Graham, and Robert Sugden. (1983). "A Rationale for Preference Reversal," American Economic Review 73, 428-432.

Loomes, Graham, Chris Starmer, and Robert Sugden. (1989). "Preference Reversal: Information Processing or Rational Non-Transitive Choice?" Economic Journal 99 (Supplement), 140-151.

Loomes, Graham, Chris Starmer, and Robert Sugden. (1991). "Observing Violations of Transitivity by Experimental Methods," Econometrica 59, 425-439.

Loomes, Graham, Peter Moffatt, and Robert Sugden. (2002). "A Microeconometric Test of Alternative Stochastic Theories of Risky Choice," Journal of Risk and Uncertainty 24, 103-130.

Loomes, Graham, Chris Starmer, and Robert Sugden. (2003). "Do Anomalies Disappear in Repeated Markets?" Economic Journal 113, C153-C166.

Luce, R. Duncan, and Peter C. Fishburn. (1991). "Rank- and Sign-Dependent Linear Utility Models for Finite First-Order Gambles," Journal of Risk and Uncertainty 4, 29-59. 
Munro, Alistair, and Robert Sugden. (2003). "On the Theory of Reference-Dependent Preferences," Journal of Economic Behavior and Organization 50, 407-428.

Prelec, Drazen. (1998). "The Probability Weighting Function,” Econometrica 66, 497-527.

Quiggin, John. (1982). "A Theory of Anticipated Utility," Journal of Economic Behavior and Organization 3, 323-343.

Savage, Leonard J. (1954). The Foundations of Statistics. New York: Wiley.

Schmidt, Ulrich, and John D. Hey. (2004). "Are Preference Reversals Errors? An Experimental Investigation," Journal of Risk and Uncertainty 29, 207-218.

Schmidt, Ulrich, and Horst Zank. (2008). "Risk Aversion in Cumulative Prospect Theory," Management Science 54, 208-216.

Segal, Uzi. (1988). "Does the Preference Reversal Phenomenon Necessarily Contradict the Independence Axiom?" American Economic Review 78, 233-236.

Slovic, Paul, Dale Griffin, and Amos Tversky. (1990). "Compatibility Effects in Judgment and Choice.” In Robin Hogarth (ed), Insights in Decision-Making. Chicago: University of Chicago Press.

Starmer, Chris. (2000). "Developments in Non-Expected Utility Theory: The Hunt for a Descriptive Theory of Choice under Risk," Journal of Economic Literature 38, 332-382.

Starmer, Chris, and Robert Sugden. (1989). "Probability and Juxtaposition Effects: An Experimental Investigation of the Common Ratio Effect," Journal of Risk and Uncertainty 2, 159-178.

Starmer, Chris, and Robert Sugden. (1998). "Testing Alternative Explanations of Cyclical Choices," Economica 65, 347-361.

Sugden, Robert. (2003). "Reference-Dependent Subjective Expected Utility,” Journal of Economic Theory $111,172-191$

Trepel, Christopher, Craig R. Fox, and Russell A. Poldrack. (2005). "Prospect Theory on the Brain? Toward a Cognitive Neuroscience of Decision under Risk," Cognitive Brain Research 23, 34-50.

Tversky, Amos, and Daniel Kahneman. (1991). "Loss Aversion in Riskless Choice: A ReferenceDependent Model," Quarterly Journal of Economics 106, 1039-1061.

Tversky, Amos, and Daniel Kahneman. (1992). "Advances in Prospect Theory: Cumulative Representation of Uncertainty," Journal of Risk and Uncertainty 5, 297-323.

Tversky, Amos, Paul Slovic, and Daniel Kahneman. (1990). "The Causes of Preference Reversal," American Economic Review 80, 204-217.

Wakker, Peter P., and Amos Tversky. (1993). "An Axiomatization of Cumulative Prospect Theory," Journal of Risk and Uncertainty 7, 147-176.

Wu, George, and Richard Gonzalez. (1996). "Curvature of the Probability Weighting Function," Management Science 42, 1676-1690.

Wu, George, and Richard Gonzalez. (1999). "Nonlinear Decision Weights in Choice under Uncertainty," Management Science 45, 74-85.

Wu, George, Jiao Zhang, and Mohammed Abdellaoui. (2005). "Testing Prospect Theory using Probability Tradeoff Consistency," Journal of Risk and Uncertainty 30, 107-131. 